\title{
Predicting Spiral Wave Stability by Personalized Electrophysiology Models
}

\author{
Cesare Corrado $^{1}$, John Whitaker ${ }^{1}$, Henry Chubb ${ }^{1}$, Steven Williams ${ }^{1}$, Matt Wright ${ }^{1}$, Jaswinder Gill ${ }^{1}$, \\ Mark O'Neill ${ }^{1}$, Steven Niederer ${ }^{1}$ \\ ${ }^{1}$ King's College London, London, United Kingdom
}

\begin{abstract}
Identifying the atrial tissue that is capable of supporting sustained re-entrant spiral wave activation patterns offers a potential ablation target for atrial arrhythmias. Current strategies for identifying this substrate require the patient to be in atrial fibrillation and require a large specialized catheter or an inverse ECG vest. We propose a novel method to personalize biophysical ionic models from standard multi-electrode catheter measurements and to predict spiral wave stability using computer simulations of a tissue region.

The developed method was applied to 5 clinical cases; the spiral wave stability was analyzed on a $5 X 5 \mathrm{~cm}^{2}$ homogeneous tissue slab and stable (2/5) and unstable self-terminating (3/5) rotors were identified.
\end{abstract}

\section{Introduction}

Atrial fibrillation (AF) is a common arrhythmia, affecting almost 2.5 million people in the US, [1] and is associated with an increased incidence of cardiovascular disease, stroke and premature death [2]. In drugrefractory patients AF is commonly treated with the radiofrequency catheter ablation [3]; however its effectiveness is moderate and many patients require multiple procedures to achieve sinus rhythm [4].

The underpinning mechanisms triggering and maintaining $\mathrm{AF}$ are not known; however a heterogeneous atrial substrate represent a favorable condition for inducing and maintaining AF, [5].

Biophysical modeling provides a formal framework that combines our understanding of atria physiology, physical constraints and patient measurements to make quantitative predictions of patient response to treatment. These models provided fundamental insight into the mechanisms that underpin arrhythmia's in the ventricle and the atria, [6], but their potential to inform clinical procedures had been limited by their inability to capture the significant variability in physiology inherent in $\mathrm{AF}$ patients.

In previous modeling studies the heart tissue was considered homogeneous; regional fibrotic regions were identified from late enhancement MRI and modeled by abruptly reducing the local conductivity [7]. This approach did not take into account of the changes on the action potential properties in the fibrotic regions and assumed that all the fibrotic regions are the same. Local physiological properties have been captured using catheter measurements together with MRI imaging, [8]. But these have not been used to directly infer biophysical model parameters.

Personalizing the local electrophysiology properties in the whole atria model will improve the model accuracy for selecting the patient and optimizing treatments [9]. In [10] the authors proposed a robust and rapid pacing protocol and a model fitting algorithm that allow to generate locally personalized computational models of the human atria in a clinical time scale. From the effective refractory period (ERP) and the conduction velocity (CV) restitutions the fitting algorithm uniquely identified the 5 parameters describing the local electrophysiology of the tissue. In this work we apply the algorithm developed in [10] to constrain the parameters of the modified MitchellSchaeffer (mMS) ionic model [11] and we predict if the tissue is capable to sustain arrhythmias. Tissue properties were predicted analyzing the spiral wave stability and the pattern of the rotor tip on a personalized 2D homogeneous tissue slab.

\section{Methods}

\subsection{Pacing protocol and activation times}

The decapolar catheter depicted in Figure 1 was placed on the roof of the left atrium (LA); LA tissue was paced from the central poles $\left(\mathrm{e}_{5}, \mathrm{e}_{6}\right)$ of the decapolar catheter depicted in Figure 1; the electrograms were measured from distal, $\left(\mathrm{e}_{1}, \mathrm{e}_{2}\right),\left(\mathrm{e}_{9}, \mathrm{e}_{10}\right)$, and proximal, $\left(\mathrm{e}_{3}, \mathrm{e}_{4}\right),\left(\mathrm{e}_{7}\right.$ , $\left.\mathrm{e}_{8}\right)$, poles in a bipolar configuration, with sampling frequency of $1 \mathrm{kHz}$ (data set 1) and $4 \mathrm{kHz}$ (data sets 
$2,3,4,5)$

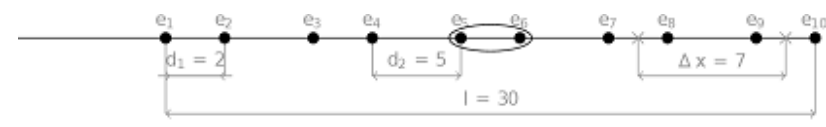

Figure 1: Decapolar catheter configuration and dimensions. The pacing stimulus is applied to the central poles, $\left(\mathrm{e}_{5}, \mathrm{e}_{6}\right)$, highlighted by the gray ellipse. The distance $\Delta \mathrm{x}$ corresponds to the distance between the barycenters of two subsequent electrode pairs.

An s1s2 protocol [12] was applied, with $\mathrm{s} 1=300,500$ and $600 \mathrm{~ms}$, (data set $1-4$ ) and $\mathrm{s} 1=300,500$ and $700 \mathrm{~ms}$ (data set 5). Before applying each premature s2 stimulus, the tissue was pre-paced with 8 stimuli of temporal interval s1 to confirm reliable capture and achieve a steady state of activity. The values of s2 started at 280, 400 and $500 \mathrm{~ms}$, respectively and were decremented by $20 \mathrm{~ms}$ down to the first $\mathrm{s} 2$ that did not present activation, identifying the ERP. The chosen pacing protocol required $<5$ min for its application.

For each bi-polar electrogram lead and for each interpacing interval the activation times were evaluated as the times corresponding to the peak of the non-linear energy operator (NLEO) [13] of the signal. A manual user check and validation on the activations was performed case by case.

\subsection{Restitution curves}

From atria electrograms it is possible to evaluate the activation time (depolarization) only, since they do not show any repolarization. From activation times only two restitution curves are directly available:

- The CV restitutions. For each premature pacing $\mathrm{s} 2, \mathrm{CV}$ is evaluated as the ratio between the distance of the barycenters of two electrode pairs and the time elapsed between the activation of each electrode pairs. The CV restitutions are evaluated for each s1 interpacing interval.

- The ERP restitutions. For each s1 inter-pacing interval, ERP represents the largest s2 not producing a propagating stimulus.

The accuracy in evaluating the ERP is proportional to the step adopted in decrementing s2. The value of $20 \mathrm{~ms}$ chosen in this work allows us to constrain the model parameters while still ensuring compatible protocol duration.

\subsection{Computational model}

Atria tissue electrophysiology was modeled with a mono-domain simplification [14] of the bi-domain model [15], when intra- and extra- cellular conductivities are considered proportional up to a constant $\lambda$. The decapolar catheter recordings were simulated with a 1D axisymmetric model of the atrial tissue, stimulated by an external current applied at the center and with a radius $\mathrm{r}_{0}=0.5 \mathrm{~mm}$, an intensity of $\mathrm{I}_{\mathrm{app}}=25 \mathrm{~ms}^{-1}$ and a duration $\mathrm{t}_{\mathrm{stim}}=0.6 \mathrm{~ms}$.

The bipolar recordings were calculated from extracellular potential differences at pairs $\left(\mathrm{e}_{7}, \mathrm{e}_{8}\right),\left(\mathrm{e}_{9}\right.$ , $\left.\mathrm{e}_{10}\right)$ and by symmetry at pairs $\left(\mathrm{e}_{1}, \mathrm{e}_{2}\right),\left(\mathrm{e}_{3}, \mathrm{e}_{4}\right)$.

The activation times were then evaluated from the computed bipolar recordings by applying the procedure described in section 2.1 and CV and ERP restitution curves were obtained as described in section 2.2.

The source term was modeled with mMS model, [11]. This model has the smallest numbers of parameters to constrain while capturing the measured CV and ERP restitution properties and it is proven to be stable to pacemaker behavior.

The mono-domain equations were discretized in space with a first order Finite Element Method (FEM) on a domain of length $\mathrm{L}=10 \mathrm{~cm}$ and with a discretization step of $d x=200 \mu \mathrm{m}$.

The model was discretized in time with the splitting algorithm presented in [16] and the solution of the cellular membrane dynamics with a backward-Euler discretization. The diffusion step was discretized with a fixed time step of $\mathrm{dt}=0.1 \mathrm{~ms}$ while the cellular membrane dynamics with a fixed time step of $\mathrm{dt}=0.01 \mathrm{~ms}$. No mass lumping was applied.

Simulated electrograms were sampled at a rate of 5 $\mathrm{kHz}$.

\subsection{Parameter fitting}

The model parameters were determined by comparing the $\mathrm{CV}$ and ERP restitution curves evaluated from clinical recordings with those of a database of pre-computed numerical simulations. The best fitting parameter set was chosen to characterize the tissue properties. A data base of candidate simulation results for 99840 combinations of the model parameters summarized in Table I was created for the pacing protocol described in section 2.1. The value of the gate potential was fixed and equal to $\mathrm{v}_{\text {gate }}=0.1$. Clinical data used in this work always displayed 1:1 capture and had an ERP $\geq 200 \mathrm{~ms}$. The parameter sets with ERP < 200ms or that failed to yield 1:1 capture. A final data set of 74933 candidates was thus obtained.

\begin{tabular}{cccccc}
\hline & $\begin{array}{c}\sigma \\
{\left[\mathrm{cm}^{2} / \mathrm{s}\right]}\end{array}$ & $\begin{array}{c}\tau_{\text {in }} \\
{[\mathrm{ms}]}\end{array}$ & $\begin{array}{c}\tau_{\text {out }} \\
{[\mathrm{ms}]}\end{array}$ & $\begin{array}{c}\tau_{\text {open }} \\
{[\mathrm{ms}]}\end{array}$ & $\begin{array}{c}\tau_{\text {close }} \\
{[\mathrm{ms}]}\end{array}$ \\
\hline $\min$ & 0.25 & 0.05 & 0.5 & 65 & 65 \\
$\max$ & 4 & 0.4 & 9.5 & 215 & 185
\end{tabular}




\begin{tabular}{rrrrrr}
\hline & $\begin{array}{c}\sigma \\
{\left[\mathrm{cm}^{2} / \mathrm{s}\right]}\end{array}$ & $\begin{array}{c}\tau_{\text {in }} \\
{[\mathrm{ms}]}\end{array}$ & $\begin{array}{c}\tau_{\text {out }} \\
{[\mathrm{ms}]}\end{array}$ & $\begin{array}{c}\tau_{\text {open }} \\
{[\mathrm{ms}]}\end{array}$ & $\begin{array}{r}\tau_{\text {close }} \\
{[\mathrm{ms}]}\end{array}$ \\
\hline step & 0.75 & 0.05 & 1 & 10 & 10 \\
\hline
\end{tabular}

Table I: Parameter values used to build the data set The parameter set that best fits clinical or simulated measurements is determined by the following two step algorithm:

- The candidate ERP restitution curve and the maximum value of $\mathrm{CV}$ on the largest $\mathrm{s} 1$ are compared against the corresponding curves for all the 74933 candidate parameter sets. A sub set of candidate parameter sets $\left(I_{1}\right)$ is identified that matches the measured ERP restitution curve and have a maximum $\mathrm{CV}$ within $20 \%$ of the recorded value.

- The $\mathrm{L}_{2}$ norms of the difference between the measured $\mathrm{CV}$ restitution curves and the $\mathrm{CV}$ restitution curves for all candidate parameter sets in set $\mathrm{I}_{1}$ are calculated and used to rank all candidate parameter sets.

\section{Results}

The algorithm was applied to 5 clinical data sets recorded from patients suffering paroxysmal AF. A unique parameter set was identified for each patient in 1 minute. In figure 2 the measured and fitted $\mathrm{CV}$ are plotted for each of the 5 clinical cases. The identified parameters are reported in Table II. To predict if these tissue properties are compatible with supporting reentrant spiral activation pattern 2D simulations of a homogeneous tissue was performed for each of the 5 clinical cases. A spiral wave was initiated with a crossfield stimulus and the pattern of the spiral tip was determined. We observed 2 distinct patterns, as depicted in figure 3: stable and breaking-up. Case 2 (not plotted) and 3 exhibited a stable pattern with a rotor tip on a fixed position; case 1, 4 and 5(not plotted) showed an unstable pattern with self-terminating rotor.

\begin{tabular}{llllll}
\hline & Case & Case & Case & Case & Case \\
& 1 & 2 & 3 & 4 & 5 \\
\hline$\sigma$ & 1.75 & 3.25 & 1.75 & 3.25 & 1.75 \\
$\tau_{\text {tn }}$ & 0.05 & 0.1 & 0.15 & 0.15 & 0.05 \\
$\tau_{\text {out }}$ & 7.5 & 2.5 & 1.5 & 2.5 & 6.5 \\
$\tau_{\text {open }}$ & 205 & 215 & 105 & 85 & 105 \\
$\tau_{\text {close }}$ & 115 & 115 & 185 & 155 & 65 \\
\hline
\end{tabular}

Table II: Estimated parameters for the 5 clinical cases
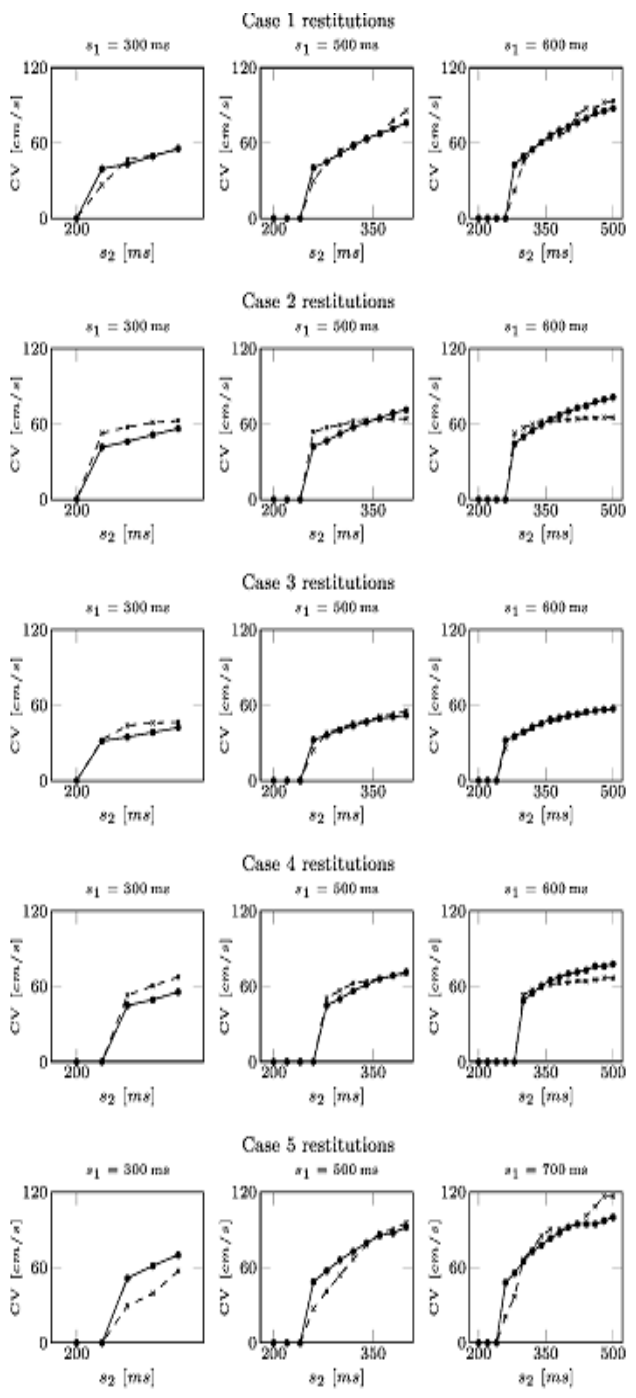

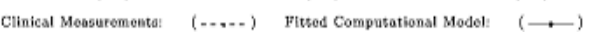

Figure 2: $\mathrm{CV}$ restitutions for the clinical cases

\section{Discussion}

In this work we applied the method proposed in [10] to 5 clinical cases from paroxysmal AF patients and we determined the local electro-physiology parameters that characterize a MS model. We predicted the pattern of the rotor tip of a spiral wave by identifying distinct stable $(2 / 5)$ and unstable self-terminating (3/5) spiral tip patterns for different cases. We characterized the ionic currents with the mMS ionic model [11]. This model was proven robust to pacemaker behavior and thus did not require any additional test on the robustness of the parameter choice. In [10] the authors adopted the standard MitchellSchaeffer model, [17] and for each case the fitting algorithm required an additional time of 5 minutes to test the robustness to pacemaker behavior. In this work, the 

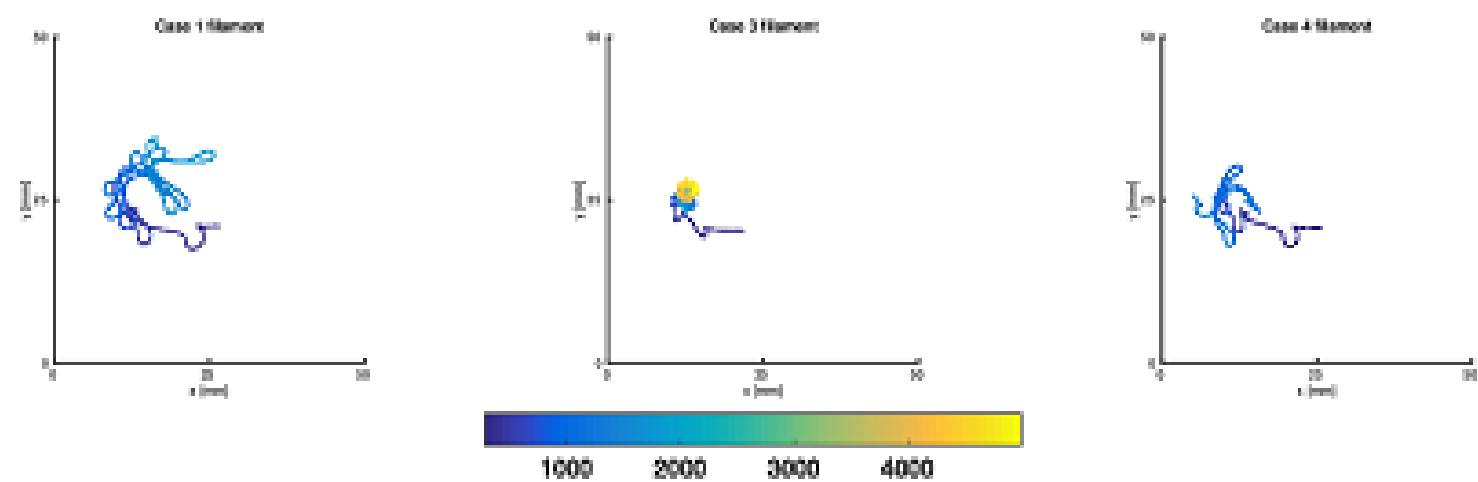

Figure 3: Rotor tip patterns for case 1, 3 and 4.

absence of spontaneous depolarizations was assured by the chosen ionic model, leading to a time required of 1 minute only to fit the parameters of each clinical case.

\section{Conclusions}

We presented a robust and clinically tractable method for the characterization of the local atrial electrophysiological properties by a phenomenological ionic cell model.

\section{Acknowledgements}

This work was supported by the British Heart Foundation (PG\13\37\30280) and the Department of Health via the National Institute for Health Research (NIHR) comprehensive Biomedical Research Centre award to Guy's \& St Thomas' NHS Foundation Trust in partnership with King's College London and King's College Hospital NHS Foundation Trust.

\section{References}

[1] Colilla S, et al. "Estimates of current and future incidence and prevalence of the atrial fibrillation in the US adult population". Am J. Cardiol, 2013; 112:1142 - 1147.

[2] Camm AJ, et al. "Guidelines for the management of atrial fibrillation". European Heart Journal, 2010;31:2369-2429.

[3] Haïsaguerre M., et al. "Electrophysiological breakthroughs from the left atrium to the pulmonary veins". Circ., 2000;102:2463-2465

[4] Cappato R, et al. "Worldwide survey on the methods, efficacy, and safety of catheter ablation for human atrial fibrillation“. Circ., 2005; 111:1100-1105.

[5] Nademane K, et al. "A new approach for catheter ablation of atrial fibrillation: mapping of the electrophysiologic substrate“. J. Am. Coll. Cardiol., 2004; 43:2044-2053.

[6] Colli Franzone P, Pavarino L, Savaré G. "Computational electrocardiology: mathematical and numerical modelling“. Complex systems in Biomedicine. Milan: Springler, 2006:187-241.
[7] McDowell KS, et al. "Methodology for patient-specific modelling of atrial fibrosis as a substrate for atrial fibrillation". J of Electrocardiology, 2012; 45:640-645.

[8] Marrouche N. F, et al., "Association of atrial tissue fibrosis identified by delayed enhancement MRI and atrial fibrillation catheter ablation: The decaaf study". J Amer Med Assoc, 2014; 311:498-506.

[9] Kneller J, et al. "Cholinergic atrial fibrillation in a computer model of a two-dimensional sheet of canine atrial cells with realistic ionic properties". Circ res, 2002; 90:e73-e87.

[10] Corrado C, et al. "Personalized models of human atrial electrophysiology derived from endocardial electrograms". IEEE Trans Biomed Eng, 2016; PP:1-1.

[11] Corrado C, Niederer S. "A two-variable model robust to pacemaker behaviour for the dynamics of the cardiac action potential". Mathematical Biosciences. 2016

[12] Murgatroyd F. "Handbook of cardiac electrophysiology: a practical guide to invasive EP studies and catheter ablation". Remedica, 2002.

[13] Kaiser JF. "On a simple algorithm to calculate 'energy' of a signal". In Acoustics, Speech and Signal Processing, 1990:381-384.

[14] Keener JP, et Sneyd J. "Mathematical physiology" Springler, 1998.

[15] Tung L. "A bi-domain model for describing ischemic myocardial D-C potentials". PhD dissertation. 1998, MIT.

[16] Either M, Bourgault Y. "Semi-implicit time discretization schemes for the bidomain model". SIAM J Numer Anal, 2008;46:2443-2468.

[17] Mitchell C, et Schaeffer DG. "A Two-Current Model for the Dynamics of Cardiac Membrane". Bull Math Bio, 2003;65:767-793

Address for correspondence.

Cesare Corrado

Department of Biomedical Engineering, King's College London. 4-th floor north wing, St. Thomas' hospital. London, SE17EK, United Kingdom

cesare.corrado@kcl.ac.uk 